\title{
Posthuman Reconstruction of the World as a Simulation in Charles Stross' Accelerando
}

\author{
Indrajit Patra (Corresponding author) \\ National Institute of Technology, Durgapur), India \\ E-mail: ipmagnetron0@gmail.com
}

Shri Krishan Rai

Department of Humanities and Social Sciences, National Institute of Technology), India

Received: 01-07-2016

Published: 02-01-2017
Accepted: 07-09-2016

doi:10.7575/aiac.ijalel.v.6n.1p.136
Advance Access Published: November 2016

URL: http://dx.doi.org/10.7575/aiac.ijalel.v.6n.1p.136

\begin{abstract}
In this paper my aim is to analyze the theme of simulation in Charles Stross' hard science fiction novel titled 'Accelerando' (2005). The discussion shall start by pointing out the enormous significance of the ideas of simulation both in the world of pure science as well as in the fields of philosophy, arts and literature. We shall show by adopting an informational approach and by using the theoretical framework of Baudrillard's 'Simulacra and Simulation' that science fiction novels like Charles Stross' 'Accelerando' treat simulation not just as an abstract mathematical entity existing independently of human beings; rather they treat simulation as an indispensable step for mankind in their journey of ascension to the Posthuman plane of existence. This paper will also try to prove that Stross' 'Accelerando' not only relies upon the description of the effects of use of many incomprehensibly powerful simulation technologies in various forms to produce the effect of estrangement upon the readers but it also implies that the very fabric of reality which deem to be real is composed of binary oppositions between different strands of contradistinctive signs and symbols which in a posthuman world will crumble together to give way to a Posthuman world replete with endless possibilities. This novel like many other contemporary hard science fiction novels implies that the reality is essentially indistinguishable from the simulation once the influence of human perception or consciousness is deducted from the equation and then the entire Universe can be interpreted as one gigantic computational entity consisting of myriads of quantum informational field operators through the manipulation of which we can derive a semblance of power indistinguishable from the actual one.
\end{abstract}

Keywords: Science Fiction, Posthumanism, Simulacra and Simulation, Quantum Nature of Creation, Portrayal of Future

\section{Significance of the Theme of Simulation and its relevance to Science Fiction}

The nature of the world in which we live in has been subject of an infinite array of speculation and hypothesis yet none has been able so far to arrive at any satisfactory conclusion. The problem might lurk in the very nature of the question or like in the act of framing the question itself since any successful inquiry into the nature of an object of reality is dependant not only upon establishing some fundamental channel of communication with the object itself so that we may be able to approach close towards chalking out any possible solution for uncovering the pattern of assemblage which gives the object its unique identity but also on our belief in the validity of numerous assumptions certain like visualizing space-time as a pre-existing and all-enveloping grid within which objects are embedded and seem to be participating in interactions among themselves thus causing events to take place. So here we are basically describing the reality as one type of manifestation which affects our senses, with which we can interact and whose nature can be described by using equations or principles deduced from and verified by numerous empirical observations and experiments and which our consciousness perceives for ourselves to be operating in a more or less predictable and comprehensible way. Now compare this against what we try to define to be Virtual. The virtual is simply the world not deemed real; it is based on fabrications, simulations, layers of artificially imposed or induced constructs and impersonations. But again the criteria for such a categorization of manifestations into two distinct categories namely the real and the virtual can be questioned by asking who determines what is best to be treated as fabrication and simulation and what is real. In the day-to-day realities consisting of phenomena best described by Newtonian mechanics one can definitely say with certainty that any object which had existed prior to its being subjected to scanning and extraction of information which then resulted in the creation of its copy can be thought of as the original whereas the latter is to be considered as a simulation or copy. But this assumption will be valid when we are thinking of our reality as something bound by and defined within the framework of an all pervasive space-time because the moment we shift our perspective to the bird's eye view of a mathematician studying the universe as a manifestation of some principles working beyond the perceptible dimensions of space-time this assumption faces serious challenges. We can with the shift of our perspective thus treat the universe as a mathematical construct or a simulated manifestation of a transcendental reality. This will make our attempts to 
categorize events into real and virtual extremely problematic. We can argue that the difference between what we currently describe to be virtual and what we are inclined to treat as real lies in the nature of characters participating in each of those worlds, for in the former the characters or player have no individual consciousness or choice of their own while in the latter the characters are very much alive, self-aware and consciously aware of himself and his surroundings. The personae in the virtual world are mere pawns of some greater power like us the human beings controlling them in a simulated environment as is evident in the case of video games and many other increasingly sophisticated applications running on VR technologies which enable us to identify ourselves with the objects and personae embedded in the pixel substrate of immersive virtual world. But according to many theories we ourselves are controlled by something much bigger than we at present imagine or are able to imagine. These theories are most often not treated seriously by mainstream physicists and so they belong to the realm of pure speculation or philosophical abstraction. There are theories which refuse to acknowledge the existence of any other controlling agent beyond this physical world, while there are some other theories which posit that either our entire universe is one giant computer which is performing calculations by itself or that all the mysteries in our universe can be deciphered by performing calculations in a systematic manner. Lee Smolin has named this kind of mathematical operations as 'Newtonian Schema' which involves quantifying and digitizing our knowledge about the fundamental realities of the universe into a certain mathematical state and then by applying some dynamic laws transforming that state into another state and finally build a faithfully simulated model of the physical universe from that computationally derived state. The question whether our universe is a simulation can also be analyzed from a different perspective like treating the entire world which we consider to be real as essentially a virtual one which proceeds from an abstract quantum reality whose reflection manifests before ourselves as physical reality and by simply residing within the physical reality it is next to impossible to tell apart reality from simulation because the entire space-time in which we reside is just a grid and it is just like the pixel substrate of any video game which makes possible for the avatars in that game to function without the avatars themselves being aware of who is controlling them and for which reason. The physics of something existing beyond that grid is also naturally expected to be different from our own with which we are trying to demystify the nature of our creation. So in case of the video games the players acting from his observer substrate position are in a driving seat or in a position to control, regulate and determine the outcomes of the entities stranded against the pixels substrate. The characters in the game are never aware of the human observer's existence and if such is the case with ours too then we can compare our endeavor to unravel the deepest secrets of our universe to that of trying to know the rules of any game if chess by simply watching the way the pieces move on the chessboard. The entire creation can be thought of as a most efficient information processing system in which the space-time fabric itself is divided into tiny fundamental, indivisible quanta much like any frame of picture is made up of countless number of pixels or any webpage is made up of strings of bits. With the arrival of quantum mechanics we are already dealing with the division and segmentation of material reality into tiniest possible bits and the ideas like Posthumanism and Transhumanism themselves are very much concerned with the classification of humans and non-humans based not on their external appearances but on the efficiency and manner of unlocking patterns of information and processing of them. As we know physics also dreams about mingling quantum with the macroscopic in the form of a 'Theory of Everything' and the first step towards the realization of it will be to determine the one fundamental building block with which space itself is made of. Since we all believe that there should definitely exist some physically meaningful minimum length beyond which the length itself cannot Lorentz contract and this minimum Universal limit of length can either be the Planck length, the length of any string particle, or the $\mathrm{TeV}$ quantum gravity length scale. The trans-Planckian region of a spacetime will always be separated from the physically observable realm of our material universe by an Event Horizon beyond which we can never hope to probe just like in Science Fiction novels the world beyond the Technological Singularity will always elude our power of prediction. So the Schwarzchild radius is that critical radius and anything having a radius less than this limit will collapse in itself to form a black hole. The Planck energy or distance scales are clearly located far beyond the grasp of our currently available technologies though introduction of String theories with additional extra dimensions compactified in a manifold brings down the energy scale from Planckian to achievable TeV scale. Alternatively we can also think of Planck's Constant as the quanta for all our space since the existence of any physically meaningful space itself necessitates the existence of energy in some form or other and the Planck's constant is the minimum amount of energy that can be associated a photon. The electric charge can also be quantized in units of multiples of the electron's charge though with the discovery of quarks with charges $\pm 1 / 3$ and $\pm 2 / 3$ the paradigm got altered but still both energy and charge can be quantized and discretized unlike the structure of the spacetime which though at the quantum level is postulated to be granular it is hard to be quantized satisfactorily in the form of a lattice composed of tiny, cellular units of Planck size. Currently available model of Quantum field theory is bound by a type of "thermodynamic" limit where lengths and distances of the order of Fermi scale are probed and investigated whereas for a more perfect understanding of the nature of reality we have to inspect it on Planck scale. However there are still many points of similarities which we still find to exist between the physical and the simulated or digital universe. One such point of similarity which we can draw between the pattern of our universe and any digital representation or a simulated reality is in the existence of Higgs field itself which currently exists in a non-zero or "on" state though not with a value as high as to make the atoms and electrons collapse. Neither does it exist in a purely " 0 " or "off" mode in which it could make the electrons massless and thus prompting them to fly apart from their atoms. So we can say that more like a digital simulation made with the help of binary codes which use the values of 0 s and $1 \mathrm{~s}$ to construct a representation of the real the Universe itself exists as a result of an interplay between the dualistic binaries operating in the form of digital representations of rest mass and energy in the value of " 1 " and representation of passive zero-energy ground state existing in the form of " 0 ". The Fermions can be represented as " 0 " because of their mutually exclusive nature while integer spin Bosons which follow 
Bose-Einstein statistics are under no obligation to follow the same sort of restriction and thus can be represented under "1". The Universe also can be thought of as a representation resulting from the supersymmetry transformations at the level of quantum mechanics where by the action of some quantum operator which is also called Supercharge the spin $1 / 2$ fermions can be transformed into bosons of spin 1 or 0 and vice versa. This clearly entails an annihilation of the boundaries separating the classical from the quantum. In another example we find that both the quarks and quasiparticles in Fractional Quantum Hall Effect have fractional electric charges and are confined within hadrons and two-dimensional systems respectively. A forcefield combines the fractional charged quarks and quasiparticles in their respective systems to manifest the sum of the fractional charges collectively as an integer. Thus here too we can denote the forbiddance of fractional charged quarks and quasiparticles to exist independently with " 0 " while the allowance of existence of electric charge as integer can be represented under the denomination " 1 " and here too we find the operation and functioning of binary dualities in the form of 0 and 1 which in turn will lead to the construction of reality at some macroscopic level. In 'accelerando' too we find that the world operates as a result of the force arising out of the interplay between two states of disallowance and allowance in which the former forbids the unaugmented, passive zeroenergy form of human being to rise to the top while encouraging the modified, augmented version of Homo Sapiens to evolve further at an extremely rapid space.

In the world of science fiction we shall also find that the simulation operates on various levels so as to make sure the annihilation of all binary oppositions so that a smooth and seamless intertwining between machine and man becomes possible. In science fiction we find ample evidences of sentient machines, omniscient extra-terrestrial intelligences making full use of the universe as a fabric composed of several layers of discretized quanta of information. They even convert the material reality into some form of computable substrate to make full use of it and this is the deconstruction of the fabric of reality to its fullest extent imaginable. This is what happens in Rudy Rucker's novel 'Postsingular' in which during the course of events which we can label under the first occurrence of a Singularity event we find that upon their arrival to Earth some nanobot-like alien machineries have slowly but steadily deconstructed every conceivable material reality by scanning and mapping them minutely and then deconstructing them atom by atom for finally building an Earth-sized system of supermassive digital computer wielding enormous raw processing power which is able to simulate almost anything existing on Earth. We also find instances when individuals are able to upload their state of consciousness in some safe haven which allows for preservation and protection of digitally uploaded states of being from which original personality of the actual persons can be reconstructed after their death. The virtual reality environment also encompasses an environment of infinite wish-fulfillment and thus can be thought of as an abode of perennial bliss. There are also numerous episodes involving the use of artificial intelligences, clones and simulated reality in its various forms which while threatening to distort the type of reality which we are currently capable of conceiving also point towards the promise of emergence of a new reality based upon an Extropian principle of mankind's ultimate deliverance through technology. 'Accelerando' tries to depict an event not as the only possible outcome of our inevitable ascendance towards the peak of progress but as some probabilistic wavefunction of myriads of possibilities which simultaneously hold in superposed state many different states of alternative realities, none of them being real till the moment of their final manifestation and realization in the form of a bump or an event perceptible as a point in the grid fabric composed of several dynamically connected and mutually intersecting nodes of spacetime. We can think of spacetime as a network built upon causal-network framework in which the events generate spacetime by their combined influence upon the fabric of spacetime and here spacetime is essentially informational. Bits and nodes of countless processing units act together to build and sustain causal nature of spacetime and science fiction novels also adopt this kind of informational approach in which the universe we deal with in the post-Singular, Posthuman era is mostly a dystopian world from our point-of-view while the action derives its impetus from the treatment of spacetime as fundamentally composed of input-output connections based on causal links. At the Planck scale, Universe can be thought of as being divided into tiny discrete units called causets which are essentially nodes of space-time connected by links of causal relationships.

In the paper entitled "Quantum theory, the Church-Turing principle and the universal quantum computer" David Deutsch has advocated for the use of Church-Turing principle as a valid physical law which can be applied to gain deeper insights into the nature of the universe as a simulation.

Here in this paper I want to prove that in Stross' 'Accelerando' a Posthuman world is conjured with the help of the ideas and concepts related to the Universe as a titanic simulation operating in a manner independent of anyone or anything and that our own life in a technologically advanced, Posthuman world will cease to be what we now term as real. These ideas will not only make us aware of the possibilities of the existence of alien intelligence or parallel universes operating on completely different physics other than our own familiar one but also open our eyes towards embracing a life where a multitude of mutually contradictory and dualistic binary oppositions will cease to pose barriers towards our path of realization of self and the true nature of reality. In the analysis of the Posthuman elements in some modern $21^{\text {st }}$ Century dystopian novels I shall be focusing here only on the theme of Simulation under the framework of Baudrillard's 'Simulacrum and Simulation' Postmodern theory.

This theory categorizes simulations into four different levels of phases where with our progress into each higher level the separation between the real and the simulation becomes even more blurry than before until the final and fourth level when the simulation becomes indistinguishable from the real. Baudrillard has also opined that the categories of science and science fiction belong to the Third-order simulacra where contrary to the popular practice of labeling science under "True" and science fiction as "parasitical simulation" we can think of these two branches as existing in a mutually reinforcing and symbiotic relationship. N. Katherine Hayles in her 'Nanoculture: Implications of the New 
Technoscience' states this relationship between science and science fiction in the following words: "the relationship of science to science fiction is not one of dichotomy but rather of imbrication and symbiosis. Science fiction infuses science and vice versa, and vectors of influence point both ways."

\section{Simulation as a Contributing Factor towards the Creation of Posthuman Scenario in Charles Stross' "Accelerando"}

As we have already stated that simulation among many other issues forms one of the most influential Posthuman elements in the modern Science Fiction where we often come across references towards the sentient intelligences building simulations that supersede and transcend life in many ways, Post-mortal state of life which is created by the use of uploaded states of individual streams of consciousness and individuals existing alongside their virtual copies or clones etc. This may appear weird and far-fetched according to the present state of advancement of science but science has often proved itself to be weirder and stranger than we can imagine from within our Newtonian framework of knowledge existing mostly within the dualism of "dynamics" (laws governing growth, development and change taking place within a system against the background of evolution of time) and "kinematics" (features or properties of motion in an object with reference to the forces causing them). Virtual reality itself as a concept is not more strange than the theories of multiverse, phenomena causing time to dilate and electromagnetic forces to slow down near supermassive bodies wielding enormous gravitational influence, objects existing in superposed states, wavefunctions collapsing as a result of experiments and many such instances when our sense bound by normal laws of ordinary mundane mechanics seem to fail to grasp the crux of the event. Physics in general attempts to solve paradoxes and problems by analyzing complex phenomena as a result of interplay of various fundamental forces but faces serious problems while analyzing the essential nature of complex systems possessing network like structure.

Science fiction novels often help us to think beyond the limitations that the present day pace of scientific advancements place upon our imaginative visualization of a distant future and it is not to be confused with mere fantasy or supernatural ride of wish fulfillment. Now the contemporary hard science fiction novels after a superficial reading might appear to not satisfy the criteria mentioned in Darko Suvin's "cognitive estrangement" which emphasizes the rational dimension of science fiction and seeks to exclude descriptions of fantastic events as mere flights of wish fulfillment as many of these hard science fiction novels deal with situations and characters for which we might find it hard to feel anything deep at all. But if we really try to understand what these contemporary hard science fiction novels try to tell us we should keep our minds open to the myriads of possibilities and unforeseen promises with which each and every step of modern scientific advancement is fraught with. It is a fact that this patch of observable universe which is 40 billion light years in diameter is but a small fraction of an infinitely large universe which is again only one among an infinite number of other universes each nucleating like a bubble in a froth of endless cosmic ocean and that not all part of this multiverse should be guided by the same fundamental laws which guide our own causal patch of creation. Also these fictional works state that to understand or predict something which is radically new or totally unforeseen we should not only be able to construct new meaning from the already existing fundamental laws governing our part of universe but also should go beyond the limitations that any of our previously held long cherished view of universe governed with the same set of rules might entail. The same rules can have different connotations once placed in different circumstances and against different backgrounds and each of our present day steps of progress though slow should not make us blind to accept the fact that the universe might not completely be what it seems - it might be after all a simulation and this simulation is indeed real depending upon what we are ready to consider as real and how far we are ready to go in our quest for uncovering the essential set of rules which we believe should help us to tell the real from the unreal. The post-Singularitarian and Posthuman elements in novels like Stross' 'Accelerando' point toward the impossibility of predicting the events of future with minute accuracy as these concepts of Posthumanism and Singularity themselves revolve around the crux of indeterminacy, indefiniteness, uncertainty and infinitude. We shall see through our discussion how the very concepts of identity formation, accumulation of knowledge and its application, incipience of life, its continuance and termination - all undergo a paradigm shift in their meaning and scope and the very fabric of our reality will give way to something inexplicably profound and thoroughly unimaginable at least with our present limitation of mind.

\section{Discussion}

Charles Stross' 'Accelerando' is a story dealing with the accelerated evolution of humanity into a posthuman state. Here we get a taste of one of the most extreme ideals of Posthumanism and Transhumanism which stresses that everything in nature is to be consumed as a raw material and only artificially constructed existence is real and meaningful. Here he centers his action around the concept of 'Technological Singularity' after the achievement of which the machines will become intelligent beyond our normal level of comprehension and unless we upgrade and modify ourselves with adequate technological augmentations and implants we will not be able to keep pace with their development. The entire novel feels like is built upon a discretized and quantified fabric of world in which information is all that matters and machines are in constant competition with man in their attempt to stay on top in the field of extracting and processing information with maximum efficiency possible. The protagonist Manfred Macx is depicted as one individual who is determined to take the singularitarian aspect of life to its very extreme by engaging in acts of ultra-advanced modes of information manipulation and retrieval, obliteration of boundaries separating the biological and the non-biological while affecting a fusion of biological and non-biological computational substrates: "Manfred doesn't believe in scarcity or zero-sum games or competition - his world is too fast and information-dense to accommodate primate hierarchy games" (Accelerando, 36). He attempts to keep himself secluded in his own sphere of "personal area network" (69) by 
uploading his memories and consciousness to some cloud-like virtual safe-house containing readily accessible streams of useful information. We find use of simulation for the purpose of storing of information too and not just for representation of information: "Subtract entropy from a data stream - coincidentally uncompressing it - and what's left is information. With a capacity of about a trillion terabytes, the suitcase's holographic storage reservoir has enough capacity to hold every music, film, and video production of the twentieth century with room to spare" (Accelerando, 52). Here we are teased to speculate upon an inherent resemblance between the holographic nature of information found in the mundane world of ours and the information encoded in the form of Universe itself. The entropy contained in any finite amount of mass-energy occupying a specified volume of space can be thought of as encoded on the boundary area or horizon of the system. Thus we can gain insight into the fundamental nature of any higher-dimensional system by measuring its holographic bound which depends mainly on the boundary area or horizon instead of the system's volume. Simulation too should be treated as a special form of holographic mode of energy or information preservation system which acts like a horizon of a system upon whose surface area many essential bits of information about the intrinsic state of the fundamentally more complex original system are encoded. There are frequent references to frequent references to some high-minded, futuristic concepts like "state vectors" which refers to the string of digits to specify the connectome and synaptic weights of a person's brain; "ghosts" or temporary instantiations of self which are created to carry out some specific task and can be made autistic to suppress some specific senses while keeping alive others for executing a particular task; "Cartesian theatre" or "sensorium" which enable one to perceive a specific part of electromagnetic spectrum while screening his other senses etc. Thus we find that man's success in maintaining his Posthuman or Transhuman state of being is dependent to a great extent upon his abilities to make use of the world around him as a source of infinite chunks of information. Baudrillard states that rapid pace of scientific and technological developments will reduce the world to mere heaps of information till all meaning is lost: “...there is more and more information, and less and less meaning" (Simulacra and Simulation, 79). Evolution of consciousness accompanies evolution of physical state of being when the latter is made to evolve according to the growing demands of the time by means of a steady fusion of technology and self. Here Simulation plays a massive role in reinforcing the digital nature of the world by its swift and almost imperceptible functioning both on the stage and from behind the curtain in bringing an end to the binary opposition between real and unreal, actual and simulation, original and the copy and the same and the other. In the first part of the novel we find the protagonist Manfred Macx is busy in creating the perfect combination of man-machine interaction through which man's central nervous system will get seamlessly connected to external machineries and computers thus facilitating in the annihilation of all boundary between the real and the virtual. Macx uploads his entire personality and memories to a virtual cloud via a series of Turing-compatible cyberware and when the cyberware gets stolen his entire personality s thrown into a cauldron of seething turmoil and unrest. In the Part 2 of the second Chapter in the novel we find the uploaded personalities of Manfred's daughter Amber Macx and 62 of her peers travel to a brown dwarf star named Hyundai +4904/-56 to find an alien router. Their entire existence is nothing more than a digital state of pure simulacra made of various strata of simulation. The mention of spacecrafts made of Computronium also reinforces the role that simulacra plays in the novel. In the novel we also find instances of biological existence taking shape out of disembodied clouds of packets of information processing operators when characters are described as arising out of pure consciousness when it is downloaded into different meatspace bodies thus forming "somatotypes" or truly physically existing identities. This and many other ideas which appear frequently tell us about the ability of future machinery and human beings to change shapes according to one's advantage or needs. We find mention of Utility fogs or nanoscale assemblage of tiny robots which are able to manipulate themselves into a continuous substance with distinctly perceptible mechanical and optical properties by mechanically rearranging themselves thus facilitating in a smooth exchange of information and energy. There are also decentralized autonomous organizations (DAO), Hypercomputation or super-Turing computation which go beyond Turing computability etc. Computronium can be used as "programmable matter," a substrate for computer modeling of virtually any real object. The simulations or the virtual realities on the most grand scale are shown as being hosted by a Computronium-made Matrioshka brain - a megastructure built around a star resembling the shape of a Dyson Sphere. According to Baudrillard, simulations are "based on information, the model, cybernetic play. Their aim is maximum operationality, hyperreality, total control." This is what happened in Ann Leckie's 'Ancillary Justice' in which the ruler of the Radch Empire Anaander Mianaai uses cybernetically enhanced ancillary soldiers to achieve maximum operationality and total control over each and every part of her Empire. In Stross' 'Accelerando' too simulacrum of power enables the technologically advanced factions to lord over the technologically less advanced ones. The Computronium-based objects are portrayed as belonging to the class of one of the most powerful instruments of simulation which can effectively recreate digital environments as modeled processes implementable by the computers.. The novel is full of images of virtual constructs embedded in hyperreal environment all of which continue to function to sustain an even greater level of simulacrum. In Baudrillard's theory of Simulacrum and Simulacra we find how with each step of our progress towards a higher order of simulacra distinction between image and representation begins to break down even further and the underlying reality is not only imitated but also replaced by an even more powerful representation than the one which preceded it. In fact the real and the material are portrayed as being destroyed and utilized for the recreation and construction of the virtual or the simulacrum as is evident in the Chapter 7 (The Curator) of Part 3 where the crew of the spaceship Field Circus comes home to find that the inner planets of the Solar System have been disassembled to build a Matrioshka brain similar to the one they encountered through the router. Thus we find that in the first phase of the novel where Macx uses his virtual reality technology and technology of programmable matter which has the ability to change its physical properties (shape, density, conductivity, optical properties, etc.) in a programmable fashion, based upon some inputs the image acts as an counterfeit of the real but the real still exists side- 
by-side with simulacrum while in the second phase the distinctions between the image and the representation begin to break down because of mass production and the proliferation of copies. In the final phase of simulation when the original human beings of flesh and blood are replaced by their cloned counterparts we find all references to the original and specialty of meaning are lost forever. Often in the novel we come across some profound questions raised in the novel like does the use of simulation to its maximum possible potentiality entail corruption and destruction of all essential characteristics which previously were used as signs to distinguish it from others? "If replicators are used to prepare a copy of a strip of bacon, right down to the molecular level, but without it ever being part of a pig, how is it to be treated? (If the mind of one of the faithful is copied into a computing machine's memory by mapping and simulating all its synapses, is the computer now a Moslem? If not, why not? If so, what are its rights and duties?)" (Accelerando, 108-109). Baudrillard rightly predicts the problematic outcome of such a situation when we will be able to simulate an object down to its each and every atom thus replicating the very essence of it for then all play of signifiers and signifieds which in normal circumstances imbue any particular object with a specific set of meanings will be cease to be meaningful themselves. So if the real can be represented as an attachment space denoted by the digit " 1 " and the simulation as a detachment space represented by the digit " 0 " the whole system of meaning can be generated as a result of a dynamic interplay between dualistic binary of " 0 " and " 1 ".

The novel also helps us to envisage the result that an onslaught from the emerging realms of simulation and nanotechnological replication technology could bring to our age-old traditional value system based on demands, differences and exchange processes: "Space (which lets you store information) and structure (which lets you process it) acquire value while dumb mass - like gold loses it. The degenerate cores of the traditional stock markets are in free fall, the old smokestack microprocessor and biotech/nanotech industries crumbling before the onslaught of matter replicators and self-modifying ideas" (Accelerando, 109). Man has achieved his dominance over other species by virtue of his superior information processing capability through which he seeks to capture, control and command different sets of information to fulfill his own needs but when he is faced to deal with an information processing agency which is able to dynamically alter each state of information at an unpredictable rate and fashion he suddenly finds his position as the central signifier seriously threatened. At its peak the simulation technology like a potent magician spins its cobweb of virtual reality in such a manner that to the mind's eye the real cease to exist as a separate and self-contained space distinct from simulation: "About ten billion humans are alive in the solar system, each mind surrounded by an exocortex of distributed agents, threads of personality spun right out of their heads to run on the clouds of utility fog - infinitely flexible computing resources as thin as aerogel - in which they live. The foggy depths are alive with high-bandwidth sparkles; most of Earth's biosphere has been wrapped in cotton wool and preserved for future examination. For every living human, a thousand million software agents carry information into the farthest corners of the consciousness address space" (Accelerando, 146). This is the moment when the whole system becomes weightless as the fundamental core of one's self is simulated to such minute details that all layers of meaning collapse on itself. The core becomes degenerate which is equivalent to reducing our belief in God to mere signs that constitute faith: "...if God himself can be simulated, that is to say can be reduced to the signs that constitute faith, then the whole system becomes weightless, it is no longer itself anything but a gigantic simulacrum - not unreal, but a simulacrum, that is to say never exchanged for the real, but exchanged for itself, in an uninterrupted circuit without reference or circumference." (Simulacra and Simulation, 6-7).

The story features not only the use of technology for creation of simulations of man and his environment but it gives us ample glimpse into various features of a posthuman world such as external minds, artificial intelligences, collective intelligences, cyberware implants, simulated human existence, living corporate entities, nanoconstruction, planetary terraforming, the singularity and almost godlike intelligences etc. Superintelligent AIs have been shown as incarnations of some of the best intellectual abilities of human beings unencumbered by the burden of decaying flesh and thus the best possible symbols of extropian ideal which can counter problems like heat-death and entropy. However the one thing that the novel maintains to be definitely beyond our horizon of understanding is envisaging the consequences of a Singularity event. In a universe built around simulacrum or virtual states of actual beings the powerful AIs "restructure the entire mass of their star system into a free-flying shell of nanocomputers, then more of them, Dyson spheres, shells within shells, like a Russian doll: a Matrioshka brain" (Accelerando, 303) and the galaxy turns into "a howling wilderness of degenerate data, fractally compressed, postconscious processes running slower and slower as they trade storage space for processing power" (Accelerando, 289). The invasion by the simulation upon real is thus can be thought of as a by-product of mankind's ever-accelerating rate of progress. Stross' 'Accelerando' also provides some insightful portrayal of various predicaments lurking in man's transition from transhumant to posthuman state. The Vile Offsprings represent the fruits of an unholy alliance between the capital and the technology and they are rightly termed as "corporate carnivores". They run a system named economics 2.0 where supply and demand equations are calculated in a pace infinitely more rapid than the traditional market system based on the "narrative chain" of personal consciousness. Economics 2.0 is unforgiving and superbly efficient and thus is able to establish influences over not just the Earth but other planets in the solar system too which are pulverized to be converted from their existence defined by "dumb matter" to spiritual essence in the form of "thinking matter". (Accelerando, 208-10) The Economics 2.0 of the Posthuman AI are so complex and merciless its functionality that even the transhumant descendants of humans are forced to flee towards the outer edge of the solar system with the fear of getting "ethnically cleansed". In the PostSingularitarian, Posthuman future the Earth orbits a dusty tunnel in space in the Solar System in which most of the solar radiation is intercepted by the concentric shells of computronium matter built from the debris of inner planets of solar system. Thus for the sake of processing huge quantities of information planets themselves have been disassembled into 
computational substrates to host endlessly accumulating bits of complex patterns of information without meaning. The solar system is thus depicted as getting reduced to a mating ground between totally meaningless signifiers and totally formless signifieds. The enormous information processing capacity of the great computronium shell has been described in the following words: "The hazy shells of computronium" are "enough to support a billion civilizations as complex as the one that existed immediately before the great disassembly" (Accelerando, 157). The crew of the 'Field Circus', a small interstellar probe using light-sail for propulsion have uploaded "themselves through its strange quantum entanglement interface for transmission to whatever alien network it connects to" (Accelerando, 160). This is an instance when the use of virtual reality has supplanted the real life itself. The uploaded self of the crew are just simulacra of their self from which their true personae can be resurrected. Again the use of quantum entanglement for teleporting self and information are also quite important as the self has been reduced to streams of data or information which can be transmitted, stored, encrypted, decrypted and retrieved at will to serve certain functions. The quantum entanglement is a mysterious faster-than-light method of communicating or transporting information between two quantum-correlated entities and the subatomic particles exchange essential bits of information using entanglement quite efficiently. The use of entire planetary systems for the purpose of making highly powerful computational processing units is accomplished by completely destroying the original nature of the planets. The very act of prescribing upper bounds to different force fields and particles energies and properties of matter imply quantization of the properties of those matter and energy fields and gravity should be no exception. So the future progress of mankind would depend upon discretization and quantization of every aspect of our material fabric of reality so that we can utilize it to maximum extent possible and like all other forces computational and information processing rates also should saturate upon attainment of an upper limit to supersede which newer forms of computational substrates and processing units need to be developed. The quantization of space-time structure or a fusion between quantum physics and gravity imply existence of certain upper limits to different quantities like for example an upper limit on the amount of energy that can be stored in any particle when the particle will be trapped in its own gravitational field is $10^{19} \mathrm{GeV}$ while the maximum physical limit to the strength of any magnetic field is $10^{57} \mathrm{G}$ and the upper limiting temperature for any physical system is $10^{32}$ degrees. From these measurements the upper limit to the power that can be emitted or generated by any physical system can also be calculated as $P_{\max } \sim \mathrm{C}^{5} / \mathrm{G} \sim 3 * 10^{59} \mathrm{ergs} / \mathrm{s}$ in which $\mathrm{C}$ is the speed of light in vacuum and $\mathrm{G}$ is the gravitational constant and this limit in turn further a universal upper limit to the information processing rate or rate of computation which is round $10^{44} \mathrm{bits} / \mathrm{sec}$. This is in fact an ultimate form of upper quantum gravity bound on the processing and propagation of any information. For a gravitating computer of the order of one solar mass maximum amount of data that can be stored is around $10^{77}$ bits while the minimal physically possible information processing rate is $\sim 1$ megabit. Thus we can see that the power that any such ultimate type of laptop or computational substrate can wield is virtually infinite as the most advanced form of computer should take full advantage of the entire memory space available which basically is one way of saying that it must efficiently convert all input matter into energy of some form. The error rate of such an efficient processing system would also generate a tremendous amount of heating for every operation performed and the thermal load of correcting errors would rival even Sun's electromagnetic output each second. So the upper limit is enormous and the planets in the solar system in Stross' 'Accelerando'are being steadily dismantled to be converted into solar-powered high-temperature nanocomputers which will in turn feed the shells of the packed layers of the Matrioshka computronium. The planets are useful only for the purpose of harvesting computronium substrate and for building a giant system of simulation operational on a cosmic scale: "The informational density of the inner planets is visibly converging on Avogadro's number of bits per mole, one bit per atom, as the deconstructed dumb matter of the inner planets (apart from Earth, preserved for now like a picturesque historic building stranded in an industrial park) is converted into computronium. And it's not just the inner system. The same forces are at work on Jupiter's moons, and those of Saturn, although it'll take thousands of years rather than mere decades to dismantle the gas giants themselves" (Accelerando, 194). The level of simulation and resimulation in the novel gets even deeper as we progress through the storyline when even the fundamental elements required to from a true identity of oneself seem to be missing as the godlike alien intelligences start simulating, resimulating, digitally storing, retrieving and transferring the identities of real individuals in an endless cycle till all points of references to the source is lost and decohered: "After experiencing your life, the weakly godlike agencies have expelled you. For reasons unknown, they chose to do this by transmitting your upload state and genome/proteome complex to receivers owned and operated by a consortium of charities based on Saturn... In summary: You are a reconstruction of someone who lived and died a long time ago, not a reincarnation. You have no intrinsic moral right to the identity you believe to be your own, and an extensive body of case law states that you do not inherit your antecedent's possessions. Other than that, you are a free individual" (Accelerando, 241).

Thus we see that a new type of techno-capitalist system has emerged as a result of an unholy interfusion between capitalism and high level technological innovation in which not only the properties, profits and assets of the individuals but their very identity faces existential crisis. The individuals are not entitled to claim their identity only by virtue of inhabiting the body of someone for he/she may not be the original possessor of the body, the body might have belonged to a whole different person altogether. The parts of one's persona that can be simulated include one's constituent units of nervous system too and as we know any form of simulation incorporates gradual but complete dismantling of the original source. This is what happens in every instance when anything is scanned thoroughly down to its individual atoms so as to extract any form of essential information that might help one to build an exact replica of the original. The nervous system state vectors are such uploaded forms of simulation: “...take a neuron, map its synapses, replace with microelectrodes that deliver identical outputs from a simulation of the nerve. Repeat for entire brain, until you've got a working map of it in your simulator" (Accelerando, 23). Amber Macx, daughter of Manfred breaks free from her 
domineering mother and ascends to become the Queen of the Ring Imperium which has successful created a sovereign country on a small, privately owned asteroid. She has arrogated herself to an endless reservoir of power and authority by her carefully maneuvered steps to tap into inexhaustible amount of mass and energy. Mass and energy on the other hand are equivalent to information which constitutes the entire space-time. So she basically has assumed command over mutually intersecting system of grids and nodes of information patterns which constitute an entire network. Her enormous power and capabilities are described in the following words: "She is the queen - the first individual to get her hands on so much mass and energy that she could pull ahead of the curve of binding technology, and the first to set up her own jurisdiction and rule certain experiments to be legal so that she could make use of the mass/energy intersection... She's by no means the first upload or partial, but she's the first gust front of the storm of power that will arrive when the arrogant ones achieve their goal of dismantling the planets and turning dumb and uninhabited mass into brainpower throughout the observable reaches of the universe" (Accelerando, 114-115). Thus we see the power and authority in a Posthuman, Post-Singularitarian era to be synonymous with manipulation of information and simulation to serve one's own purposes. Simulation can obliterate distinctions between real and unreal, true and false, life and death and as such can be thought of as an ethereal medium through which power and authority propagate. The dangers hidden beneath the task of unlocking infinite raw processing power by converting dumb masses into computronium substances are also hinted at. The simulation which not only hides or masks reality but decoheres, dissipates, disrupts and destroys it consists of many carefully constructed layers of simulations which mimick the real to such an unimaginable level of accuracy that one does not feel the need for reality anymore. Powerful operating systems virtualize each and every aspect of physics operating on physical universe while proving timely sensory inputs into the consciousness of the individuals experiencing the simulation so as to erase all chance for reality to take shape from the subjective experiences of those persons concerned. The persons who are placed in the immersive environment of simulation have been migrated right out of their own state of original consciousness "using an amazing combination of nanotechnology and electron spin resonance mapping" and they are now being run as "software in an operating system designed to virtualize multiple physics models and provide a simulation of reality that doesn't let them go mad from sensory deprivation!" (Accelerando, 136) The novel is full of images of virtual constructs embedded in hyperreal environment and functioning from within it to sustain an even deeper level of simulacrum. Here all distinctions between real and unreal get easily lost. In fact the real and the material are portrayed as being destroyed and utilized for the recreation and construction of the virtual or the simulacrum as is evident in the Chapter 7 (The Curator) of Part 3 where the crew of the spaceship Field Circus comes home to find that the inner planets of the Solar System have been disassembled to build a Matrioshka brain. Different versions of one single individual lead different lives across different parts in a Solar system and Transhuman or Posthuman versions of once-human beings steadily deconstruct every planetary objects for building immensely powerful computronium machines: "Beings that remember being human plan the deconstruction of Jupiter, the creation of a great simulation space that will expand the habitat available within the solar system. By converting all the nonstellar mass of the solar system into processors, they can accommodate as many human-equivalent minds as a civilization with a planet hosting ten billion humans in orbit around every star in the galaxy" (Accelerando, 147). Humans though, as has been stated in the novel are not genetically hardwired to survive in most of the simulation conditions existing elsewhere either in different parts of our Universe or in other Verses and in some incredibly complex world the humans will be nothing more than petty worms and parasites squirming through the dead and decaying bodies in the battlefield in search of meaning and relevance: "Humans can't survive in most of the simulation spaces here. Universes with physics models that don't support our kind of neural computing" (Accelerando, 179). Death also loses its meaning in the Posthuman era filled with hyperreal elements and simulation technologies which can virtually store any data of a being while it is still existing so that it can retrieve and recuperate the original subject from that stored data when cease to occupy any physically meaningful space. Death has been equated here more or less with the collapse of a wavefunction which till its arrival upon a particular point of destination exists as an ensemble of many superposed states. This ensemble of multitude of probabilities corresponds to what we can term the "quantum potentia" which in an observer-participant measurement event will be converted into the "quantum actua". Here we can actually see how during an experiment or measurement an infinite number of microscopic electronpositron quantum operator fields undergo spontaneous CPT symmetry breaking through its interaction with an observer via a medium filled with photons acting as packets of an operator field. This is what happens in an Universe like ours where entropy always increases universally and this can be thought of as a phenomenon associated with the emission of causal radiation arrow or clouds of photons from a composite made of fermions. This is what is termed as death from anthropic point-of-view which simply means an increase of entropy in the arrangement of atoms for a particular biological organism and from the extropian perspective associated with the Post-singularitarian science fiction novum this irreversible process of increase of entropy with the passage of time can occasionally be countered by perpetuating the flow of one's consciousness in an unbroken stream: "You don't need to believe in God to believe in embedded realities. We use them every day, as mind tools. Apply anthropic reasoning and isn't it clear that our entire universe is probably a simulation? We're living in the early epoch of the universe. Probably this is but a simulation in some ancient history engine's panopticon, rerunning the sum of all possible origins of sentience, a billion trillion megayears down the line. Death will be like waking up as someone bigger, that's all" (Accelerando, 209). The "sum of all possible origins of a sentience" reminds us of Feynman's sum-over-paths (or sum-over-histories) approach which states that in its journey from a point A to another point B the particle traverses probably an infinite number of different paths and each of these paths will have a probability amplitude associated with it and the ensemble of all these probabilities can be thought of as the particle's wavefucntion. In the sum-over-all-possible-paths approach the spreading of the waveform of the particle will initiate a process of cancelling out or reinforcing of different wave patterns of various amplitudes till the 
only path that is left after the end of such interference is what the particle actually follows. Human existence is too conceived in a form like that where death and birth are associated with collapse of probabilistic wave patterns and adoption of a specific state against the background of an undivided spacetime continuum. We can imagine human existence as moving toward an ultimately unattainable form of true "signified" and that our self much like our language is full of fissures, voids, gaps and opposing forces yielding irreconcilable tension. It is structured more like a non-linear narrative of labyrinthine structure in which we come across brief, occasional moments providing us with opportunities to transcend past the diving barrier to arrive at a higher plane of existence. Such a plane of existence will be the Posthuman state of being but the arrival at which will imply annihilation of innumerable forms of oppositions, contradictions and false vacuums. Our Universe itself is in a state of metastability which can be conceived as a false vacuum in which different sector of more "true" bubble could expand at speed of light. A false vacuum could at any time tunnel to a more stable lower energy configuration in the form of a true vacuum and this could spell disaster for our universe. A bubble of true vacuum could expand from any corner of Universe and could collide with our false vacuum state of universe thus toppling its particles, forces and to a completely different quantum state. This is much like how a stable Posthuman condition could arise as a result of the collapse of our mundane Human existence in which the fissures, gaps, breaks and voids in our unaugmented state of living would grow and transform themselves into abiding realities through the use of Simulation and manipulation of information patterns while destroying in the process the existing signs, structures and binary realities which make up our reality.

\section{Conclusion}

This paper deals with the question of reality as is represented in Charles Stross' 'Accelerando' and this vision of reality is reverberated in several other contemporary hard science fiction novels in which reality in a Posthuman or PostSingular world is represented as something which is inconceivably complex, indefinitely enigmatic and bizarrely terrifying. In this age only those who will most successfully manipulate and control information patterns will be able to wield power and supremacy while others will serve under them. The emergence of the sentient machines in the postSingular era will relegate humans into obscurity unless the humans radically alter themselves with implants, cybernetic enhancements and other scientific aids and appendages. The fundamental pattern in which the basic building blocks of our Universe is arranged will be revealed to us as something made up of grids and nodes arranged in mutually intersecting lines inside a network-like structure which is spacetime itself. Man's or his Posthuman counterpart's relentless obsession with tweaking with harnessing more computational power might drive him to deconstruct the very fabric of material reality by transforming ordinary matters into computronium substances. The culture, economics, technological progress and the very definition of human life will all be propelled and finally radically altered by some infinitely more advanced form of machine intelligences than what we encounter at present. They will become man's master and not mere accessories like what they are at present and man will act like an extension of this highly enhanced machine intelligence. But it will be hard to label this condition either as a pure dystopia or a pure utopia as the same world can be perceived and interpreted in radically different ways from different viewpoints and as such the same world can be a dystopia to some groups of people while an utopia to many others who will be cut off and sequestrated from the bliss of scientific and technological advancements. Also all forms of binary oppositions and interplay of signs will be rendered obsolete and useless and we will come across signifiers without signifieds, signs without core, subjects without objects, information without meaning and simulations without originals. A new type for reality will dawn before our eyes and the gaps, inconsistencies and vacuums which were previously held in suppression under the rigid, logocentric, traditional discourse will become powerful enough to assert themselves and subvert the currently existing world order. The Posthuman condition thus can be conceived as a more efficient, lower energy and stable state which demand less resource for sustaining mutually irreconcilable dualistic oppositions between various opposing ideas and concepts. The main demand of this age will be centered round the use of matter for computational purposes which in turn will be utilized for the creation and maintenance of a gigantic simulation indistinguishable from reality.

\section{References}

Stross, Ch. (2006). Accelerando. 2005. New York: Ace-Penguin.

Baudrillard, J. (1976). L'Echange symbolique et la mort. Paris: Gallimard.

---. (2009). Simulacra and Simulations. Trans. Sheila Fraser. Ann Arbor: University of Michigan Press, 1994. Print.

Rucker, Rudy. Postsingular. 2007. New York: Tor.

Kurzweil, R. (1999). The Age of Spiritual Machines: When Computers Exceed Human Intelligence. New York: Penguin.

---. (2005). The Singularity is Near. New York: Penguin.

Hayles, N. (1991). Katherine, ed. Chaos and Order: Complex Dynamics in Literature and Science. Chicago: U of Chicago P.

---. (1999). How We Became Posthuman: Virtual Bodies in Cybernetics, Literature, and Informatics. Chicago: U of Chicago P.

Kellner, D., Baudrillard, J. (1989). From Marxism to Postmodernism and Beyond. Stanford, CA: Stanford University Press. 

Vinge, V. (1993). "The Coming Technological Singularity: How to Survive in the Post-Human Era" Vision-21: Interdisciplinary Science and Engineering in the Era of Cyberspace NASA-CP-10129, 1993.

Suvin, D. (1979). Metamorphoses of Science Fiction: On the Poetics and History of a Literary Genre. Yale University Press, 1979.

Lloyd, S. (2006). Programming the Universe. A Quantum Computer Scientist Takes On the Cosmos Alfred A. Knopf.

Tegmark, M. (2002). The Mathematical Universe," in Visions of Discovery: Shedding New Light on Physics and Cosmology, R. Chiao, Ed. Cambridge: Cambridge Univ. Press.

Bostrom, N. (2002). "Are you Living in a Computer Simulation?," Philosophical Quarterly, vol. 53, pp. 243-255.

Tegmark, M. (1997). "The interpretation of Quantum Mechanics: Many Worlds or Many Words," in arXiv:quant-ph/9709032v1 vol. 15 Sep.

Deutsch, D. (1985). "Quantum theory, the Church-Turing principle and the universal quantum computer", Proceedings of the Royal Society (London) 400: 97-117, 1985. doi:10.1098/rspa.1985.0070 